\title{
CHARACTERIZATION OF COLLISION KERNELS
}

\author{
Laurent Desvillettes $^{1}$ And Francesco Salvarani ${ }^{2}$
}

\begin{abstract}
In this paper we show how abstract physical requirements are enough to characterize the classical collision kernels appearing in kinetic equations. In particular Boltzmann and Landau kernels are derived.
\end{abstract}

Mathematics Subject Classification. 76P05.

Received: May 2, 2002. Revised: January 7, 2003.

\section{INTRODUCTION}

Kinetic equations (in particular those in which appears a collision kernel) are usually derived (at the formal level) from Hamiltonian systems. While for linear equations it is in general possible to give a rigorous proof of those derivations ( $c f$. for example [9] for the linear Boltzmann equation, [6] and [8] for the linear Landau equation), nonlinear equations give rise to very difficult problems, and rigorous results exist only for special regimes (local (in time) solutions for the Boltzmann equation (cf. $[4,14]$ ), or global (in time) small solutions of the Boltzmann equation $(c f .[12,13])$ ). When singular (nonlinear) kernels are concerned (non cutoff Boltzmann kernel, or Landau kernel), the derivation of the corresponding kinetic equation seems completely open, though the equations themselves have been extensively studied ( $c f$. for example $[1,2,7]$ and the references therein for recent results on these equations).

We wish to derive here such singular nonlinear kernels by solving a quite different (and much simpler) problem. We look for kernels satisfying given properties such as Galilean invariance and existence of equilibrium states, and prove that they have to be the kernels we wish to derive (such as cutoff or non cutoff Boltzmann or Landau kernels). In other terms we derive the kernels of the kinetic equations but not the equations themselves.

This line of ideas has already been followed in $[3,15]$. We propose here a systematic treatment for binary collisions in a mono-atomic (non quantum and non relativistic) rarefied gas.

Let $Q$ be the kernel under investigation. The requirements for $Q$ will be the following: $Q$ is continuous (in a sense to be specified), bilinear, invariant with respect to Galilean transformations, it takes the value 0 on the set of Maxwellian (that is, Gaussian) functions, and it preserves the non-negativity of the solutions of the corresponding evolution equation.

In order to show how our method works on a more tractable problem, we shall also consider the case of a kernel $L$ (in a periodic 1D context) which is linear, continuous, invariant under translations and parity, taking

\footnotetext{
Keywords and phrases. Boltzmann, Landau, collision kernels.

1 École Normale Supérieure de Cachan, Centre de Mathématiques et leurs Applications, 61 Avenue du Président Wilson, 94235 Cachan, France. e-mail: desville@cmla.ens-cachan.fr

2 Università degli Studi di Pavia, Dipartimento di Matematica, Via Ferrata, 1, 27100 Pavia, Italy.

e-mail: salvarani@dimat.unipv.it
} 
the value 0 on the constant functions, and conserving the non-negativity (of the solution of the related evolution equation).

The assumptions of the nonlinear case and the corresponding conclusions are given in a precise way in the following theorem:

Theorem 1. Let

$$
Q: \mathcal{S}\left(\mathbb{R}^{n}\right) \times \mathcal{S}\left(\mathbb{R}^{n}\right) \rightarrow C_{\text {temp }}^{\infty}\left(\mathbb{R}^{n}\right)=C^{\infty}\left(\mathbb{R}^{n}\right) \cap \mathcal{S}^{\prime}\left(\mathbb{R}^{n}\right)
$$

be an operator such that:

(1) $Q$ is bilinear and continuous from $\mathcal{S}\left(\mathbb{R}^{n}\right) \times \mathcal{S}\left(\mathbb{R}^{n}\right)$ to $C_{\text {temp }}^{\infty}\left(\mathbb{R}^{n}\right)$;

(2) $Q$ is invariant under translations, that is $Q \circ \tau_{h}=\tau_{h} \circ Q$ for all $h \in \mathbb{R}^{n}$ (where $\tau_{h} \varphi(x)=\varphi(x+h)$ );

(3) $Q$ is invariant under rotations (and orthogonal symmetries), that is $Q \circ \Gamma_{R}=\Gamma_{R} \circ Q$, where $\Gamma_{R} \varphi(x)=$ $\varphi(R x)$, for all $R \in O(n, \mathbb{R})$;

(4) $Q\left(M_{u, T}, M_{u, T}\right)=0$, where $M_{u, T}$ is the Maxwellian function of arbitrary mean velocity $u \in \mathbb{R}^{n}$ and temperature $T>0$ :

$$
M_{u, T}(v)=\frac{1}{(2 \pi T)^{n / 2}} \mathrm{e}^{-|v-u|^{2} / 2 T} ;
$$

(5) for all $f, g \in \mathcal{S}\left(\mathbb{R}^{n}\right), v_{0} \in \mathbb{R}^{n}$, such that $f, g \geq 0$ on $\mathbb{R}^{n}$ and $f\left(v_{0}\right)=0$, one has $Q(f, g)\left(v_{0}\right) \geq 0$.

Then the operator $Q$ is a Boltzmann-Landau operator in the following sense:

$$
Q(f, g)(v)=\langle K(x, y, v), f(x) g(y)\rangle_{x, y},
$$

where $K$ is a distribution of the form

$$
K(x, y, v)=\delta_{|x-y|=|x+y-2 v|} \operatorname{Pf}\left(\frac{\mu\left(\left|\frac{x+y}{2}-v\right|, \arccos \left(\frac{x+y-2 v}{|x+y-2 v|} \cdot \frac{x-y}{|x-y|}\right)\right)}{1-\frac{x+y-2 v}{|x+y-2 v|} \cdot \frac{x-y}{|x-y|}}\right),
$$

and where $\mu$ is a positive measure which is even and $2 \pi$-periodic in the second variable.

Note that the standard form of the Boltzmann-Landau operator (that is, the sum of a (non-cutoff) Boltzmann operator and a Landau operator, $c f$. [5] and the references therein for example) is easily obtained from equation (1) by making changes of variables.

We detail those changes of variables in dimension 2. Defining the variables $\rho \in \mathbb{R}_{+}, \theta \in \mathbb{R} / 2 \pi \mathbb{Z}$ and $v_{*} \in \mathbb{R}^{2}$ by

$$
\begin{aligned}
& x=\frac{v+v_{*}}{2}+\rho R_{\theta}\left(\frac{v-v_{*}}{2}\right), \\
& y=\frac{v+v_{*}}{2}-\rho R_{\theta}\left(\frac{v-v_{*}}{2}\right),
\end{aligned}
$$


we get

$$
\begin{aligned}
Q(f, g)(v)= & \left\langle\delta_{\rho=1} \operatorname{Pf}\left(\frac{\mu\left(\frac{\left|v-v_{*}\right|}{2}, \theta\right)}{1-\cos \theta}\right) \rho\left|v-v_{*}\right|^{2}, f\left(\frac{v+v_{*}}{2}+\rho R_{\theta}\left(\frac{v-v_{*}}{2}\right)\right)\right. \\
& \left.\times g\left(\frac{v+v_{*}}{2}-\rho R_{\theta}\left(\frac{v-v_{*}}{2}\right)\right)\right\rangle_{\rho, \theta, v_{*}} \\
= & \left\langle P f\left(\frac{\mu\left(\frac{\left|v-v_{*}\right|}{2}, \theta\right)}{1-\cos \theta}\right)\left|v-v_{*}\right|^{2}, f\left(\frac{v+v_{*}}{2}+R_{\theta}\left(\frac{v-v_{*}}{2}\right)\right)\right. \\
& \left.\times g\left(\frac{v+v_{*}}{2}-R_{\theta}\left(\frac{v-v_{*}}{2}\right)\right)\right\rangle_{\theta, v_{*}} .
\end{aligned}
$$

This is the usual form of the Boltzmann-Landau operator.

Note that the Landau part of the operator corresponds to the atomic part of $\mu$ in $(\cdot, 0)$, whereas if $\mu=\theta^{2} \nu$, one recovers the formula for the "regular" Boltzmann operator:

$$
\begin{aligned}
& Q(f, g)(v)=\int_{v_{*} \in \mathbb{R}^{2}} \int_{\theta \in]-\pi, \pi[} {\left[f\left(\frac{v+v_{*}}{2}+R_{\theta}\left(\frac{v-v_{*}}{2}\right)\right)\right.} \\
&\left.\times g\left(\frac{v+v_{*}}{2}-R_{\theta}\left(\frac{v-v_{*}}{2}\right)\right)-f(v) g\left(v_{*}\right)\right]\left|v-v_{*}\right|^{2} \frac{\theta^{2}}{1-\cos \theta} \mathrm{d} \nu\left[\frac{\left|v-v_{*}\right|}{2}, \theta\right] .
\end{aligned}
$$

We now comment assumptions 1 to 5 .

The first part of assumption 1 (bilinearity of the operator) is related to the fact that only binary collisions are taken into account, and that no quantum effects such as Pauli's exclusion principle are assumed.

The second part of assumption 1 is not directly related to physics. It is a very weak assumption of continuity which allows to eliminate "pathological" kernels. Note that the continuity from $C_{c}\left(\mathbb{R}^{n}\right) \times C_{c}\left(\mathbb{R}^{n}\right)$ to $C\left(\mathbb{R}^{n}\right)$ would be a much stronger assumption, which would not be satisfied by classical kernels such as Landau's kernel or the non cutoff Boltzmann kernel.

Assumptions 2 and 3 express the Galilean invariance. Their replacement by the invariance with respect to the Lorentz group should lead to the relativistic collision kernels.

Assumption 4 ensures that the Maxwellian functions are equilibria for our kernel. The choice of different functions (such as the Fermi-Dirac) could lead to different kernels (such as the Boltzmann kernel with Pauli exclusion term).

Assumption 5 means, through the maximum principle, that for any $g \geq 0, f_{0} \geq 0$, the solution $f$ of

$$
\partial_{t} f=Q(f, g), \quad f(0)=f_{0}
$$

should remain nonnegative. We use however this formulation since we want to work only on the operator and not on the equation (which is not necessarily well posed under the rather not stringent conditions of smoothness imposed by assumption 1).

In Section 2, we deal with a simpler problem, in a linear context. Then, we prove Theorem 1 in Section 3. Finally, we give in Section 4 a typical counterexample.

\section{THE LINEAR CASE}

In order to show how our ideas work, we start with a brief study of a much simpler problem, namely that of the linear Boltzmann kernel in a periodic setting. 
We prove in this section the

Theorem 2. Let $C_{p}^{\infty}(\mathbb{R})$ be the space of $C^{\infty}, 2 \pi$-periodic functions defined on $\mathbb{R}$, and

$$
L: C_{p}^{\infty}(\mathbb{R}) \rightarrow C_{p}^{\infty}(\mathbb{R})
$$

be an operator such that:

(1) $L$ is linear and continuous from $C_{p}^{\infty}(\mathbb{R})$ to $C_{p}^{\infty}(\mathbb{R})$;

(2) $L$ is invariant under translation, that is $L \circ \tau_{h}=\tau_{h} \circ L$ for all $h \in \mathbb{R}$;

(3) $L$ is invariant under parity, that is $L \circ P=P \circ L$, where $P$ is the parity operator: $P \varphi(x)=\varphi(-x)$;

(4) $L 1=0$;

(5) for all $f \in C_{p}^{\infty}(\mathbb{R})$ and $x_{0} \in \mathbb{R}$ such that $f \geq 0$ and $f\left(x_{0}\right)=0$, one has $(L f)\left(x_{0}\right) \geq 0$.

Then the operator $L$ can be written as

$$
L f(x)=\int \frac{f(x+y)-f(x)-\sin y f^{\prime}(x)}{\sin ^{2} y} \mathrm{~d} \mu(y)+\lambda f^{\prime \prime}(x),
$$

where $\lambda \geq 0, \mu$ is a nonnegative $2 \pi$-periodic and even measure such that $\mu(\{0\})=0$, and the integral is taken over a period.

The converse statement also holds: the operator $L$ defined above satisfies assumptions 1 to 5 .

Proof. We begin by writing the operator $L$ in integral form. This can be done thanks to a variant of Schwartz' kernel theorem ( $c f .[11]$, p. 128):

Proposition 3. To each linear continuous map $L$ from $C_{p}^{\infty}(\mathbb{R})$ to $C_{p}^{\infty}(\mathbb{R})$ corresponds a (unique) kernel $K \in$ $\mathcal{D}_{p}^{\prime}(\mathbb{R} \times \mathbb{R})$ (space of $2 \pi$-periodic distributions with respect to both variables) such that

$$
\langle L f, \varphi\rangle=\langle K, \varphi \otimes f\rangle
$$

for all $f, \varphi \in C_{p}^{\infty}(\mathbb{R})$.

We now translate assumptions 2 to 5 in this formalism. It is clear that $K$ satisfies

2 .

$$
K(x+h, y+h)=K(x, y) \quad \text { for all } \quad h \in \mathbb{R},
$$

3 '.

$$
K(-x,-y)=K(x, y)
$$

4 '.

$$
\langle K, \varphi \otimes 1\rangle=0 \quad \text { for all } \quad \varphi \in C_{p}^{\infty}(\mathbb{R}),
$$

5 .

$$
\left(\sin \left(y-x_{0}\right)\right)^{2} K\left(x_{0}, y\right) \geq 0 .
$$

Note that for 5 , we use the fact that $\left(\sin \left(\cdot-x_{0}\right)\right)^{2} \varphi$ is a nonnegative function taking the value 0 at point $x_{0}$ as soon as $\varphi$ is nonnegative.

Thanks to assumption 2', we can find $k$ in $\mathcal{D}_{p}^{\prime}(\mathbb{R})$ such that

$$
K(x, y)=k(x-y)
$$


and assumptions 3' to 5' become

3".

$4 "$

$$
k(-x)=k(x)
$$

5".

$$
\langle k, 1\rangle=0
$$

$$
(\sin (\cdot))^{2} k \geq 0
$$

We then use the (classical)

Proposition 4. Let $k \in \mathcal{D}_{p}^{\prime}(\mathbb{R})$ be such that $(\sin (\cdot))^{2} k \geq 0$. Then

$$
k(x)=\operatorname{Pf}\left(\frac{\mu(x)}{(\sin x)^{2}}\right)+a \delta_{0}(x)+b \delta_{0}^{\prime}(x),
$$

where $\mu$ is a nonnegative $2 \pi$-periodic measure, and $a, b \in \mathbb{R}$.

Here, since we are in the context of $2 \pi$-periodic distributions, $\delta_{0}, \delta_{0}^{\prime}$ denote the $2 \pi$-periodicized versions of the Dirac mass at 0 and its derivative. Moreover, the finite part $\operatorname{Pf}\left(\frac{\mu(x)}{(\sin x)^{2}}\right)$ is defined by the formula

$$
\left\langle P f\left(\frac{\mu(x)}{(\sin x)^{2}}\right), \varphi\right\rangle=\int \frac{\varphi(x)-\varphi(0)-\sin x \varphi^{\prime}(0)}{(\sin x)^{2}} \mathrm{~d} \mu(x),
$$

the integral being taken on one period.

Proof. We use the decomposition

$$
\langle k, \varphi\rangle=\langle k, 1\rangle \varphi(0)+\langle k, \sin x\rangle \varphi^{\prime}(0)+\left\langle(\sin x)^{2} k(x), \frac{\varphi(x)-\varphi(0)-\sin x \varphi^{\prime}(0)}{(\sin x)^{2}}\right\rangle,
$$

and denote $\mu(x)=(\sin x)^{2} k(x), a=\langle k, 1\rangle$ and $b=-\langle k, \sin x\rangle$. But this is a nonnegative distribution, and therefore a nonnegative measure.

Using Proposition 4, we get

$$
k(x)=\operatorname{Pf}\left(\frac{\mu(x)}{(\sin x)^{2}}\right)+a \delta_{0}(x)+b \delta_{0}^{\prime}(x) .
$$

Assumption 4" ensures that $a=0$.

Then, assumption 3" gives $b=0$ and $\mu(x)=\mu(-x)$. Indeed, assumption 3" implies that for all $\varphi \in C_{p}^{\infty}(\mathbb{R})$,

$$
\int \frac{\varphi(x)-\varphi(0)-\sin x \varphi^{\prime}(0)}{(\sin x)^{2}}[\mathrm{~d} \mu(x)-\mathrm{d} \mu(-x)]=2 b \varphi^{\prime}(0) .
$$

Then, for any $\psi \in C_{p}^{\infty}(\mathbb{R})$, we use $\varphi(x)=(\sin x)^{2} \psi(x)$, and get

$$
\int \psi(x)[\mathrm{d} \mu(x)-\mathrm{d} \mu(-x)]=0 .
$$

Therefore, $\mu(x)=\mu(-x)$ and $b=0$.

Writing $\mu=2 \lambda \delta_{0}+\nu$ with $\nu(\{0\})=0$, we get the final form of operator $L$ :

$$
L f(x)=\int \frac{f(x+y)-f(x)-\sin y f^{\prime}(x)}{(\sin y)^{2}} \mathrm{~d} \nu(y)+\lambda f^{\prime \prime}(x) .
$$




\section{Proof of the Main theorem}

We now turn to the proof of Theorem 1. We follow the guidelines of the linear case. Since we are interested in quadratic operators, we need a characterization theorem for such objects. The following lemma is a direct consequence of [10] (Chap. 1, Th. 7, p. 17):

Lemma 1. Let $Q$ be a continuous bilinear operator from $\mathcal{S}\left(\mathbb{R}^{n}\right) \times \mathcal{S}\left(\mathbb{R}^{n}\right)$ to $C_{\text {temp }}^{\infty}\left(\mathbb{R}^{n}\right)$. Then, there exists a (unique) kernel $K \in \mathcal{S}^{\prime}\left(\mathbb{R}^{n} \times \mathbb{R}^{n} \times \mathbb{R}^{n}\right)$ such that for all $f, g, \varphi \in \mathcal{S}\left(\mathbb{R}^{n}\right)$,

$$
\langle Q(f, g), \varphi\rangle=\langle K, f \otimes g \otimes \varphi\rangle
$$

We now use assumptions 2 to 5 of Theorem 1 and Lemma 1 . They imply that

2 .

$3^{\prime}$

$$
K(x+h, y+h, v+h)=K(x, y, v) \quad \text { for all } \quad h \in \mathbb{R}^{n},
$$

4 .

$$
K(R x, R y, R v)=K(x, y, v) \quad \text { for all } \quad R \in O(n, \mathbb{R})
$$

$$
\left\langle K(x, y, v), M_{u, T}(x) M_{u, T}(y)\right\rangle_{x, y}=0
$$

5 .

$$
|x-v|^{2} K(x, y, v) \geq 0
$$

In order to get 5', it is enough to observe that thanks to 5 , for all nonnegative functions $f, g$ of $\mathcal{S}\left(\mathbb{R}^{n}\right)$ and all $v \in \mathbb{R}^{n}$, one has

$$
\left\langle K(x, y, v),|x-v|^{2} f(x) g(y)\right\rangle_{x, y} \geq 0 .
$$

Thanks to assumption 2', there exists $k \in \mathcal{S}^{\prime}\left(\mathbb{R}^{n} \times \mathbb{R}^{n}\right)$ such that

$$
K(x, y, v)=k(x-v, y-v) .
$$

Then, assumptions 3' to 5' become

$3 "$.

$$
k(R x, R y)=k(x, y) \quad \text { for all } \quad R \in O(n, \mathbb{R}),
$$

$4 "$.

$5 "$

$$
\left\langle k, M_{u, T} \otimes M_{u, T}\right\rangle=0
$$

$$
|x|^{2} k(x, y) \geq 0
$$

We now can perform the change of variables (of Jacobian $2^{n}$ ):

$$
C:(a, b) \rightarrow(a-b, a+b)
$$

and consider the distribution $k_{1}=k \circ C$. Assumptions 3" to 5" become

$3 "$ '.

$4 "$,

$$
k_{1}(R a, R b)=k_{1}(a, b) \quad \text { for all } \quad R \in O(n, \mathbb{R}) \text {, }
$$

$$
\left\langle k_{1}(a, b), \mathrm{e}^{-\lambda|b|^{2}-\lambda|a-u|^{2}}\right\rangle=0 \quad \text { for all } \quad \lambda>0, u \in \mathbb{R}^{n},
$$

$5 \%$.

$$
|a-b|^{2} k_{1}(a, b) \geq 0
$$


Lemma 2. If $F \in \mathcal{S}^{\prime}\left(\mathbb{R}^{n}\right)$ and

$$
\left\langle F, \mathrm{e}^{-\beta|\cdot-u|^{2}}\right\rangle=0
$$

for all $\beta>0$ and $u \in \mathbb{R}^{n}$, then $F=0$.

Proof. We note that

$$
\left\langle F * \mathrm{e}^{-\beta|\cdot-u|^{2}}\right\rangle=\left(F * \mathrm{e}^{-\beta|\cdot|^{2}}\right)(u),
$$

so that for all $\beta>0, F * \mathrm{e}^{-\beta|\cdot|^{2}}=0$. We conclude by letting $\beta$ go to infinity.

Lemma 3. If $F \in \mathcal{S}^{\prime}\left(\mathbb{R}^{n}\right)$ and

$$
\left\langle F, \mathrm{e}^{-\beta|x|^{2}}\right\rangle=0
$$

for all $\beta>0$, then $\langle F, \phi\rangle=0$ for all radial function $\phi$ in $\mathcal{S}\left(\mathbb{R}^{n}\right)$.

Proof. Note that it is enough to prove this result for $F \in \mathcal{S}\left(\mathbb{R}^{n}\right)$, since the property verified by $F$ is stable by convolution and multiplication by any (centered) Gaussian function.

Then, we take the derivative of the formula $\left\langle F, \mathrm{e}^{-\beta|x|^{2}}\right\rangle=0$ with respect to $\beta$, and get that for all $k \in \mathbb{N}$, $\beta>0,\left\langle F,|x|^{2 k} \mathrm{e}^{-\beta|x|^{2}}\right\rangle=0$. It remains to use the density of polynomials in $L^{2}\left(\mathbb{R}_{+}\right)$with respect to the measure $r^{n-1} \mathrm{e}^{-\beta r^{2}} \mathrm{~d} r$ to conclude.

We denote in the sequel by $(\mathbb{R} / 2 \pi \mathbb{Z})_{e}$ the space corresponding to a variable which gives rise to $2 \pi$-periodic even functions or distributions on $\mathbb{R}$. We now define the distribution $l \in \mathcal{D}^{\prime}\left(\mathbb{R}_{*}^{+} \times \mathbb{R}_{*}^{+} \times(\mathbb{R} / 2 \pi \mathbb{Z})_{e}\right)$ by the formula

where

$$
\langle l, \psi\rangle=\left\langle k_{1}, \phi\right\rangle
$$

$$
\phi(a, b)=|a|^{1-n}|b|^{1-n} \psi\left(|a|,|b|, \arccos \left(\frac{a}{|a|} \cdot \frac{b}{|b|}\right)\right) .
$$

Thanks to assumption $3 "$, it is possible to recover $k_{1}$ from $l$. Namely,

$$
\left\langle k_{1}, \phi\right\rangle=\langle l, \psi\rangle,
$$

with

$$
\psi(\alpha, \beta, \theta)=|\alpha|^{n-1}|\beta|^{n-1} \iint_{|\omega|=1,|\sigma|=1, \omega \cdot \sigma=\cos \theta} \phi(\alpha \omega, \beta \sigma) .
$$

Note that, by taking this definition, we just gave a precise characterization of a distribution $l$ verifying $k_{1}(a, b)=$ $l\left(|a|,|b|, \arccos \left(\frac{a}{|a|} \cdot \frac{b}{|b|}\right)\right)$.

Assumptions 4"' and 5"' now imply that $4 "$ ".

$5 "$ "

$$
\left\langle l\left(\rho_{1}, \rho_{2}, \theta\right), 1\right\rangle_{\theta}=0
$$

$$
\left(\rho_{1}^{2}+\rho_{2}^{2}-2 \rho_{1} \rho_{2} \cos \theta\right) l\left(\rho_{1}, \rho_{2}, \theta\right) \geq 0 .
$$

In order to get 4 " ", we note that thanks to Lemmas 2 and $3,\left\langle k_{1}, \phi\right\rangle=0$ for all functions $\phi$ of $\mathcal{S}\left(\mathbb{R}^{n} \times \mathbb{R}^{n}\right)$ which are radially symmetric with respect to the second variable. Then, thanks to formula (3), we get that $\langle l, \psi\rangle=0$ for all functions $\psi$ of $\mathcal{D}\left(\mathbb{R}_{*}^{+} \times \mathbb{R}_{*}^{+} \times(\mathbb{R} / 2 \pi \mathbb{Z})_{e}\right)$ which do not depend on the last (angular) variable. But this exactly means $4 ", "$. As for $5 "$, , it is directly obtained by setting $\rho_{1}=|a| ; \rho_{2}=|b| ; \theta=\arccos \left(\frac{a}{|a|} \cdot \frac{b}{|b|}\right)$.

We then define $L_{1}$ on $\mathcal{D}^{\prime}\left(\mathbb{R}_{*}^{+} \times \mathbb{R}_{*}^{+} \times(\mathbb{R} / 2 \pi \mathbb{Z})_{e}\right)$ by

$$
L_{1}\left(\rho_{1}, \rho, \theta\right)=l\left(\rho_{1}, \rho_{1} \rho, \theta\right)
$$


Properties 4"" and 5"" become

$$
4 ", " \text {. }
$$

$$
\left\langle L_{1}\left(\rho_{1}, \rho, \theta\right), 1\right\rangle_{\theta}=0
$$

5"',

$$
\left(1+\rho^{2}-2 \rho \cos \theta\right) L_{1}\left(\rho_{1}, \rho, \theta\right) \geq 0 .
$$

We now prove the following lemma:

Lemma 4. The support of $L_{1}$ is included in the set $\{\rho=1\}$.

Proof. This lemma is fully proven if we show that $\left\langle L_{1}, \varphi\right\rangle=0$ for all test-functions $\varphi$ vanishing on a neighbourhood of the set $\{\rho=1\}$. For this reason, we consider a nonnegative function $\Psi\left(\rho_{1}, \rho\right)$ of $\operatorname{class} C^{\infty}$ such that $\Psi\left(\rho_{1}, \rho\right)=0$ for all $\rho \in(1-\varepsilon, 1+\varepsilon), \varepsilon>0$, and a nonnegative $\chi \in C_{p}^{\infty}$. Then,

$$
\left\langle L_{1}\left(\rho_{1}, \rho, \theta\right), \Psi\left(\rho_{1}, \rho\right) \chi(\theta)\right\rangle_{\rho_{1}, \rho, \theta}=\left\langle\left(1+\rho^{2}-2 \rho \cos \theta\right) L_{1}\left(\rho_{1}, \rho, \theta\right), \frac{\Psi\left(\rho_{1}, \rho\right)}{1+\rho^{2}-2 \rho \cos \theta} \chi(\theta)\right\rangle_{\rho_{1}, \rho, \theta} .
$$

Now, since $\Psi\left(\rho_{1}, \rho\right)=0$ in a neighborhood of $\{\rho=1\}$, the function $\Psi\left(\rho_{1}, \rho\right) /\left(1+\rho^{2}-2 \rho \cos \theta\right)$ is still of class $C^{\infty}$ and then, by $5 "$, ,

Moreover, 4""' guarantees also that

$$
\left\langle L_{1}\left(\rho_{1}, \rho, \theta\right), \Psi\left(\rho_{1}, \rho\right) \chi(\theta)\right\rangle_{\rho_{1}, \rho, \theta} \geq 0 .
$$

$$
\left\langle L_{1}\left(\rho_{1}, \rho, \theta\right), \Psi\left(\rho_{1}, \rho\right) \otimes 1_{\theta}\right\rangle_{\rho_{1}, \rho, \theta}=0 .
$$

If we consider the distribution of $\mathcal{D}^{\prime}\left((\mathbb{R} / 2 \pi \mathbb{Z})_{e}\right)$ :

$$
T=\left\langle L_{1}\left(\rho_{1}, \rho, \theta\right), \Psi\left(\rho_{1}, \rho\right)\right\rangle_{\rho_{1}, \rho},
$$

the previous conditions imply that $T \geq 0$ and $\langle T, 1\rangle_{\theta}=0$.

This implies that $T=0$, since $T$ is nonnegative and of mass 0 . This in turn ensures that

$$
\left\langle L_{1}\left(\rho_{1}, \rho, \theta\right), \Psi\left(\rho_{1}, \rho\right) \otimes \chi(\theta)\right\rangle=0
$$

for all $\chi \in C_{p}^{\infty}$, so that $\operatorname{supp}\left(L_{1}\right) \subset\{\rho=1\}$.

The previous lemma allows us to apply a variant of a theorem characterizing the distributions whose support is contained in a plane (see [11], Th. 2.3.5).

Theorem 5. Let $x=\left(x^{\prime}, x^{\prime \prime}\right)$ be a splitting of the variables in $\left(\mathbb{R} \text { or }(\mathbb{R} / 2 \pi \mathbb{Z})_{e}\right)^{n}$ in two groups. If $u$ is a distribution in $\left(\mathbb{R} \text { or }(\mathbb{R} / 2 \pi \mathbb{Z})_{e}\right)^{n}$ of order $k$ (with compact support for a non periodic variable) contained in the plane $x^{\prime}=0$, then

$$
u(\phi)=\sum_{|\alpha| \leq k} u_{\alpha}\left(\phi_{\alpha}\right)
$$

where $u_{\alpha}$ is a distribution of compact support and order $k-|\alpha|$ in the $x^{\prime \prime}$ variables, $\alpha=\left(\alpha^{\prime}, 0\right)$ and

$$
\phi_{\alpha}\left(x^{\prime \prime}\right)=\left.\partial^{\alpha} \phi\left(x^{\prime}, x^{\prime \prime}\right)\right|_{x^{\prime}=0} .
$$

We consider for a given nonnegative $\psi \in \mathcal{D}\left(\mathbb{R}_{+}^{*}\right)$ the distribution $W_{\psi}(\rho, \theta)=\left\langle L_{1}\left(\rho_{1}, \rho, \theta\right), \psi\left(\rho_{1}\right)\right\rangle$ of $\mathcal{D}^{\prime}\left(\mathbb{R}_{+}^{*} \times\right.$ $\left.(\mathbb{R} / 2 \pi \mathbb{Z})_{e}\right)$. Note that for all $\psi$, the distribution $W_{\psi}$ has a compact support in $\rho$ (depending on $\psi$ ), so that we can apply Theorem 5 , and get

$$
W_{\psi}(\rho, \theta)=\sum_{i=0}^{m} W_{i}(\theta) \otimes \delta_{\rho=1}^{(i)}
$$


where $W_{i} \in \mathcal{D}^{\prime}\left((\mathbb{R} / 2 \pi \mathbb{Z})_{e}\right)$. Therefore assumptions 4 "', and $5 "$ "', respectively become

$$
\left\langle\sum_{i=0}^{m} W_{i}(\theta) \otimes \delta_{\rho=1}^{(i)}, 1_{\theta}\right\rangle_{\theta}=0
$$

and

$$
\left(1+\rho^{2}-2 \rho \cos \theta\right) \sum_{i=0}^{m} W_{i}(\theta) \otimes \delta_{\rho=1}^{(i)} \geq 0 .
$$

Thanks to equation (4), if we choose test-functions of type $\psi(\rho)=(\rho-1)^{j} \Phi(\rho)$, where $\Phi$ is a given test function of $\mathcal{D}\left(\mathbb{R}^{+}\right)$which is equal to 1 in a neighborhood of $\rho=1$, we obtain a triangular system of equations on the quantities $\left\langle W_{i}(\theta), 1_{\theta}\right\rangle_{\theta}$, whose solution is

$$
\left\langle W_{i}(\theta), 1_{\theta}\right\rangle_{\theta}=0, \quad i=0, \ldots, m
$$

On the other hand, equation (5) becomes after computation (when $m \geq 2$ ):

$$
\begin{aligned}
& \sum_{i=0}^{m-2}\left[2(1-\cos \theta) W_{i}(\theta)-\right.\left.2(i+1)(1-\cos \theta) W_{i+1}(\theta)+(i+2)(i+1) W_{i+2}(\theta)\right] \otimes \delta_{\rho=1}^{(i)} \\
&+\left[2(1-\cos \theta) W_{m-1}(\theta)-2 m(1-\cos \theta) W_{m}(\theta)\right] \otimes \delta_{\rho=1}^{(m-1)} \\
&+\left[2(1-\cos \theta) W_{m}(\theta)\right] \otimes \delta_{\rho=1}^{(m)} \geq 0 .
\end{aligned}
$$

We now apply the distribution defined above to functions of type

$$
\psi^{*}(\rho, \theta)=\left(1-A(1-\rho)^{m}+\frac{A^{2}}{4}(1-\rho)^{2 m}\right) s(\theta)
$$

for all $A \in \mathbb{R}$, where $s$ is an arbitrary nonnegative function.

The function $\lambda(\rho)=1-A(1-\rho)^{m}+\left(A^{2} / 4\right)(1-\rho)^{2 m}$ has the following derivatives at $\rho=1$ :

$$
\lambda(1)=1, \quad \lambda^{(i)}(1)=0 \quad \text { for } i=1, \ldots, m-1 \quad \lambda^{(m)}(1)=(-1)^{m+1} A m ! .
$$

It is clear that $\lambda, \psi^{*} \geq 0$, and therefore we get

$$
\left\langle 2(1-\cos \theta) W_{0}(\theta)-2(1-\cos \theta) W_{1}(\theta)+2 W_{2}(\theta), s(\theta)\right\rangle+(-1)^{2 m+1} 2 A m !\left\langle(1-\cos \theta) W_{m}(\theta), s(\theta)\right\rangle \geq 0 .
$$

But this is true for all $A \in \mathbb{R}$, so that

$$
\left\langle(1-\cos \theta) W_{m}(\theta), s(\theta)\right\rangle=0
$$

Note that (8) still holds when $m=1$ although (7) is slightly different in this case (no term in $W_{2}$ appears).

Since $s$ is arbitrary (nonnegative), we get for $m \geq 1$,

$$
(1-\cos \theta) W_{m}(\theta)=0
$$

We now need the following lemma:

Lemma 5. Let $W \in \mathcal{D}^{\prime}\left((\mathbb{R} / 2 \pi \mathbb{Z})_{e}\right)$ satisfy the equations $(1-\cos \theta) W=0$ and $\langle W, 1\rangle=0$. Then $W=0$.

Proof. Since $(1-\cos \theta) W=0$, we know that $W=a \delta_{0}+b \delta_{0}^{\prime}$ (the Dirac masses here are taken in the sense of $2 \pi$-periodicized distributions). But $W$ is even, so that $b=0$. Finally, $a=0$ because $\langle W, 1\rangle=0$. 
Thanks to Lemma $5,(6)$ and (9), we see that for $i \geq 1, W_{i}=0$.

Then, thanks to (5), we get $(1-\cos \theta) W_{\psi}=(1-\cos \theta) W_{0} \geq 0$, so that $(1-\cos \theta) W_{\psi}$ is a measure with its support in $\{\rho=1\}$. Therefore, we get $(1-\cos \theta) W_{\psi}=\delta_{\rho=1} \mu_{\psi}(\theta)$ for some nonnegative $2 \pi$-periodic even measure $\mu_{\psi}$. But this implies that $(1-\cos \theta) L_{1}\left(\rho_{1}, \rho, \theta\right)$ is nonnegative and has its support included in $\{\rho=1\}$, so that

$$
(1-\cos \theta) L_{1}\left(\rho_{1}, \rho, \theta\right)=\delta_{\rho=1} \mu\left(\rho_{1}, \theta\right)
$$

for some nonnegative measure $\mu$ which is $2 \pi$-periodic and even with respect to its second variable.

As a consequence, we see that

$$
L_{1}\left(\rho_{1}, \rho, \theta\right)=\delta_{\rho=1}\left\{\operatorname{Pf}\left(\frac{\mu\left(\rho_{1}, \theta\right)}{1-\cos \theta}\right)+\alpha \delta_{\theta=0}+\beta \delta_{\theta=0}^{\prime}\right\} .
$$

But $L_{1}$ is even with respect to the third variable, so that $\beta=0$, and $\left\langle L_{1}, 1\right\rangle_{\theta}=0$, so that $\alpha=0$. Finally, we get

$$
L_{1}\left(\rho_{1}, \rho, \theta\right)=\delta_{\rho=1} \operatorname{Pf}\left(\frac{\mu\left(\rho_{1}, \theta\right)}{1-\cos \theta}\right) .
$$

Recalling the relation between $L_{1}$ and $K$, we exactly get formula (1).

\section{Counterexample}

In this section, we discuss the choice of property 5 of Theorem 2, which deals with the positivity of the operator.

Another natural condition would indeed be

6. for all $f \in C_{p}^{\infty}(\mathbb{R}),\langle L f, f\rangle \leq 0$.

Note however that

$$
L f(x)=\int-\cos (x-y) f(y) \mathrm{d} y
$$

defines a kernel satisfying 1, 2, 3, 4 and 6 , but which is not of the form given in the conclusion of Theorem 2 (it does not satisfy condition 5 ).

In fact a simple Fourier analysis of the problem shows that

$$
L f(x)=\int k(x-y) f(y) \mathrm{d} y
$$

defines a kernel satisfying $1,2,3,4$ and 6 as soon as

$$
c_{0}(k)=0, \quad c_{m}(k)=c_{-m}(k) \leq 0 \quad \text { for all } \quad m \in \mathbb{N}^{*} .
$$

In the nonlinear case, the analogue of assumption 6 would be Boltzmann's H-theorem. It seems however difficult to produce counterexamples analogous to (10) in such a situation.

Acknowledgements. F.S. acknowledges the GNFM of the Italian National Institute of Higher Mathematics (INDAM) for partial support. Both authors acknowledge the EU financed network No. HPRN-CT-2002-00282. We finally wish to thank Prof. Claude Bardos and François Golse for pointing out to us the interest of the problem studied here. 


\section{REFERENCES}

[1] R. Alexandre, L. Desvillettes, C. Villani and B. Wennberg, Entropy dissipation and long-range interactions. Arch. Ration. Mech. Anal. 152 (2000) 327-355.

[2] R. Alexandre and C. Villani, On the Landau approximation in plasma physics. To appear in Ann. I.H.P. An. non linéaire.

[3] A.V. Bobylev, The Boltzmann equation and the group transformations. Math. Models Methods Appl. Sci. 3 (1993) $443-476$.

[4] C. Cercignani, R. Illner and M. Pulvirenti, The mathematical theory of dilute gases. Springer Verlag, New York (1994).

[5] L. Desvillettes, Boltzmann's kernel and the spatially homogeneous Boltzmann equation. Riv. Mat. Univ. Parma 6 (2001) $1-22$.

[6] L. Desvillettes and V. Ricci, A rigorous derivation of a linear kinetic equation of Fokker-Planck type in the limit of grazing collisions. J. Statist. Phys. 104 (2001) 1173-1189.

[7] L. Desvillettes and C. Villani, On the spatially homogeneous Landau equation for hard potentials. Part I: Existence, uniqueness and smoothness. Comm. Partial Differential Equations 25 (2000) 179-259.

[8] D. Dürr, S. Goldstein and J. Lebowitz, Asymptotic motion of a classical particle in a random potential in two dimensions: Landau model. Comm. Math. Phys. 113 (1987) 209-230.

[9] G. Gallavotti, Rigorous theory of the Boltzmann equation in the Lorentz gas. Nota interna No. 358, Istituto di Fisica, Università di Roma (1973).

[10] I.M. Guelfand and N.Y. Vilenkin, Les distributions, Tome IV, Applications de l'analyse harmonique. Dunod, Paris (1967).

[11] L. Hörmander, The analysis of linear partial differential operators I. Springer Verlag, Berlin (1983).

[12] R. Illner and M. Pulvirenti, Global validity of the Boltzmann equation for a two-dimensional rare gas in the vacuum. Comm. Math. Phys. 105 (1986) 189-203.

[13] R. Illner and M. Pulvirenti, Global validity of the Boltzmann equation for two- and three-dimensional rare gas in the vacuum: erratum and improved result. Comm. Math. Phys. 121 (1989) 143-146.

[14] O. Lanford, Time evolution of large classical systems. Springer Verlag, Lecture Notes in Phys. 38 (1975) 1-111.

[15] R.W. Preisendorfer, A mathematical foundation for radiative transfer. J. Math. Mech. 6 (1957) 685-730.

To access this journal online: www.edpsciences.org 